\title{
The influence of Pauli blocking effects on the properties of dense hydrogen
}

\author{
W. Ebeling ${ }^{1}$, D. Blaschke ${ }^{2,3}$, R. Redmer ${ }^{4}$, H. Reinholz ${ }^{4,5}$, \\ G. Röpke ${ }^{4}$ \\ ${ }^{1}$ Institute of Physics, Humboldt University, 12489 Berlin, Germany, \\ ${ }^{2}$ Institute for Theoretical Physics, University of Wroclaw, 50-204 Wroclaw, Poland, \\ ${ }^{3}$ Bogoliubov Lab. for Theoretical Physics, JINR Dubna, 141980 Dubna, Russia, \\ ${ }^{4}$ Institute of Physics, University of Rostock, 18051 Rostock, Germany, \\ ${ }^{5}$ School of Physics, University of Western Australia, Crawley, 6009 WA, Australia.
}

\begin{abstract}
We investigate the effects of Pauli blocking on the properties of hydrogen at high pressures, where recent experiments have shown a transition from insulating behavior to metal-like conductivity. Since the Pauli principle prevents multiple occupation of electron states (Pauli blocking), atomic states disintegrate subsequently at high densities (Mott effect). We calculate the energy shifts due to Pauli blocking and discuss the Mott effect solving an effective Schrödinger equation for strongly correlated systems. The ionization equilibrium is treated on the basis of a chemical approach. Results for the ionization equilibrium and the pressure in the region $4000 \mathrm{~K}<T<$ $20000 \mathrm{~K}$ are presented. We show that the transition to a highly conducting state is softer than found in earlier work. A first order phase transition is observed at $T<6450$ $\mathrm{K}$, but a diffuse transition appears still up to $20000 \mathrm{~K}$.
\end{abstract}

\section{Introduction}

The physical properties of dense hydrogen are a topic of high interest, in particular the transition of hydrogen to a highly conducting phase, which is considered a type of Mott transition. Here, we will consider the effects of Pauli's exclusion principle on high-density hydrogen plasmas. The previous studies of dense hydrogen included several hypothetical assumptions about the character of the high-density phase [1, 2, 3, 4, 5, 6, 7). While metallization of solid hydrogen near $T=0 \mathrm{~K}$ has not been clearly verified so far for pressures of up to $300 \mathrm{GPa}$ [8], metal-like features have been observed in shockcompression [8, 9, 10]. Metal-like conductivities have been observed around $140 \mathrm{GPa}$ and $3000 \mathrm{~K}$ [9]. Recent experiments were able to reach that region as well and to provide detailed information on the equation of state (EoS) and the conductivity in the Mbar-region [11]. The transition to metal-like behavior changes drastically our present understanding of the behavior of hydrogen at ultra-high pressures. In the present paper, we will show that the most important effect leading to the destruction of bound states is Pauli blocking. Due to the Pauli exclusion principle, the free electrons in the plasma cannot penetrate into the interior of atoms and molecules. At high densities this leads 
to an enormous pressure acting on the neutrals which will finally be ionized. The effective energy levels of hydrogen, which strongly depend on density and weakly on temperature, are introduced into the thermodynamic functions. In order to calculate the ionization/dissociation equilibrium we minimize the free energy with respect to the composition. Recently, we derived an expression for the free energy of dense hydrogen $F_{\mathrm{H}}$ [12, 13, 14] in the framework of the chemical picture and calculated the degree of ionization $\alpha$ and the degree of dissociation $\beta$ as well as the isothermal EoS, the hugoniots and the isentropes. Pauli blocking was taken into account by the concept of excluded volume, which is based on the idea of space occupation by atoms and molecules. We will show that a more fundamental approach based on an effective Schrödinger equation [15, 16], leads to important modifications of the earlier results.

\section{Effective Schrödinger equation and bound states of pairs}

We focus on the interaction between atoms and free electrons and present a microscopic treatment based on the underlying Pauli exclusion principle. In the following we will use Rydberg units with $m_{e}=1 / 2, \hbar=1, e^{2} / 4 \pi \epsilon_{0}=2$, so that the binding energies of the isolated hydrogen atom are simply $E_{n}^{0}=-1 / n^{2}$. Embedding the hydrogen atom in a plasma environment, the interactions with the medium are treated by an effective wave equation [2, 15, 16]

$$
p^{2} \psi_{n}(p)-\sum_{q} V(q) \psi_{n}(p+q)+\sum_{q} H^{\mathrm{pl}}(p, q) \psi_{n}(p+q)=E_{n} \psi_{n}(p),
$$

where $V(q)=8 \pi / q^{2}$ denotes the Fourier transform of the Coulomb potential. The center of mass motion has been neglected, assuming the adiabatic limit $m_{e} / m_{p} \ll 1$. In general, the plasma Hamiltonian $H^{\mathrm{pl}}(q)$ will depend also on $\vec{P}$ and on the energy, if dynamical and retardation effects are taken into account. The plasma Hamiltonian will shift the energy eigenvalues $E_{n}=E_{n}^{0}+\Delta E_{n}$ and will modify the wave functions $\psi_{n}(p)$. In particular, due to the plasma interaction the binding energies may merge with the continuum so that the bound states disappear, if the influence of the plasma increases with increasing density. This dissolution of bound states is called Mott effect and has important consequences for the macroscopic properties of the plasma. Let us evaluate now the mean-field energy shift of bound states writing the effective Hamiltonian of pairs in a plasma as

$$
H^{\mathrm{pl}}(p, q)=\sum_{q^{\prime}} f_{e}\left(p+q^{\prime}\right)\left[V(q) \delta\left(q^{\prime}\right)-V\left(q^{\prime}\right) \delta(q)\right]
$$

$f_{e}(p)=1 /\left(\exp \left[\beta\left(p^{2} / 2 m-\mu\right)\right]+1\right)$ is the Fermi distribution. Within first order, the shift of the energy eigenvalues is obtained with the unperturbed wave functions $\phi_{n}(p)$ as

$$
E_{n}-E_{n}^{0}=\sum_{p, p^{\prime}} \phi_{n}^{*}(p) V\left(p^{\prime}-p\right) f_{e}(p) \phi_{n}\left(p^{\prime}\right)-\sum_{p, p^{\prime}} \phi_{n}^{*}(p) V\left(p^{\prime}-p\right) f_{e}\left(p^{\prime}\right) \phi_{n}(p) .
$$

The first term in Eq. (3) is the Pauli shift which is due to Pauli blocking, and can be rewritten by inserting the Schrödinger equation as

$$
\Delta E_{n}^{\text {Pauli }}=\sum_{p} \phi_{n}^{*}(p)\left(p^{2}-E_{n}^{0}\right) f_{e}(p) \phi_{n}(p) .
$$


A simple expression is found in the low-temperature and low-density limit, where the Fermi distribution with the normalization $\sum_{p} f_{e}(p)=n_{e} / 2$ is concentrated near $p=0$. In the zero temperature limit, we have a Fermi sphere with Fermi momentum $p_{F}=\left(3 \pi^{2} n_{e}\right)^{1 / 3}$. The energy shift of the ground state is then

$$
\Delta E_{1}^{\text {Pauli }}=\frac{1}{2} n_{e}\left(-E_{1}^{0}\right)\left|\phi_{1}(0)\right|^{2}=32 \pi n_{e} .
$$

At intermediate temperatures we may approximate the Fermi distribution by a Boltzmann distribution and find

$$
\Delta E_{1}^{\text {Pauli }}(T)=32 \pi n_{e} G\left(T / T_{0}\right) \simeq 32 \pi n_{e} /\left[1+(77 / 16) T / T_{0}\right],
$$

where $G(x)=\left\{\sqrt{x}(1+1 / x)-\sqrt{\pi}\left(1-x-x^{2} / 4\right)[1-\operatorname{erf}(1 / \sqrt{x})] \exp (1 / x)\right\} / x^{7 / 2}$, see also [17], and $T_{0}=1 \mathrm{Ryd} / k_{\mathrm{B}}=157886 \mathrm{~K}$ is the ionization temperature. Similarly, the Fock term as the second term in Eq. (3) can be evaluated as

$$
\Delta E_{1}^{\text {Fock }}=-128 n_{e} \int_{0}^{\infty} d p \frac{1}{\left(1+p^{2}\right)^{4}}=-20 \pi n_{e} .
$$

It compensates partially the Pauli shift. Since the temperature dependence of the Fock shift is similar to that of the Pauli shift we may use in first approximation the same temperature function as in (6) and the total shift is approximated by

$$
\Delta E_{1}^{\text {Fock }}+\Delta E_{1}^{\text {Pauli }}=12 \pi n_{e} G\left(T / T_{0}\right) .
$$

The shift is shown in Fig. 1, dashed line, indicating a rather steep increase of the bound state energy with density. Due to phase space occupation, the ground state energy is shifted and may merge with the continuum of scattering states, indicating the dissolution of bound states. Considering in Eq. (2) the continuum part of the spectrum describing scattering states, only the Fock shift contributes to the energy shift. The lowest energy in the continuum occurs at $p=0$ and is shifted by $\Delta E^{\text {Fock }}(p=0)=-\sum_{q} V(q) f_{e}(q)=-4 p_{F} / \pi=-4\left(3 n_{e} / \pi\right)^{1 / 3}$. However, the two-particle continuum state can only be created at the Fermi momentum since all states below that are occupied. Thus the continuum of scattering states begins at $p_{F}$ where we have in the zero temperature limit the Fock shift

$$
\Delta E^{\text {Fock }}\left(p_{F}\right)=-\sum_{p} V\left(\vec{p}-\vec{p}_{F}\right) f_{e}(p)=-2 p_{F} / \pi=-2(3 / \pi)^{1 / 3} n_{e}^{1 / 3},
$$

shown also by the dashed line in Fig. 1, Extrapolating the low-density results to higher densities, the ground state disappears in first approximation at a density $n_{e} \simeq 0.015$. This corresponds to an average distance of $r_{0} \simeq 2 a_{B}$ and is below the Mott criterion. The Mott condition $r_{0} \simeq a_{B}$ with $(4 \pi / 3) n_{e} r_{0}^{3}=1$ expresses the idea that atoms are destroyed if the mean distance of the electrons is equal or smaller than the Bohr radius. There exist many alternative estimates of the binding energy shift. For example, the confined atom model [2, 19] assumes that the atom is embedded into a sphere with radius $r_{0}$. In first approximation this theory gives the shift (in Rydberg units) $\Delta E_{1}^{\mathrm{ca}}=\pi^{2} r_{0}^{-2}$. Correspondingly, the binding energy would disappear at $r_{0} \simeq 3 a_{B}$, i.e., already at a much smaller density (see also Fig. 1). Better estimates based on numerical solutions of the Schrödinger equation give a value of about $r_{0} \simeq 2 a_{B}$ [19]. Our estimate is in the same region. 


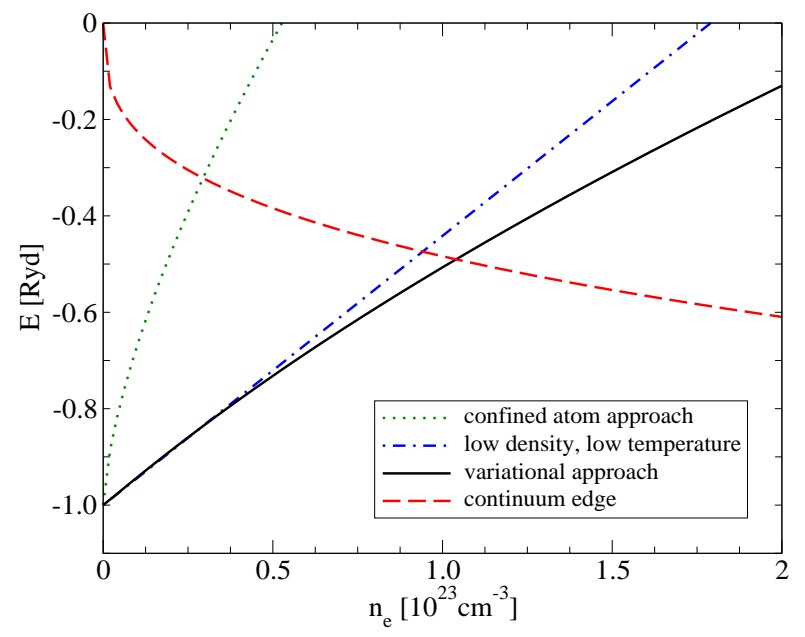

Figure 1. Density dependence of the effective ground state energy of hydrogen. The low-temperature estimate (dash-dotted line) according to Eq. (8) is compared with the confined atom approach (dotted line). The solid line corresponds to the variational approach. We have shown also the lowering of the continuum edge (dashed line) according to Eq. (9).

\section{Evaluation of the mean-field energy shift by variational approach}

According to our estimate, the effective binding energy would disappear at $n_{e} \simeq 10^{-2}$. This seems to be too early; the reason is that perturbation approximations tend to overestimate effects. Better results may be obtained by variational approximations to the solution of the effective Schrödinger equation. To apply the Ritz variational approach, we have to symmetrize the Hamiltonian in the effective wave equation introducing the function $\Psi_{n}(p)=\psi_{n}(p)\left[1-f_{e}(p)\right]^{-1 / 2}$. We will consider the zero temperature case and use the ansatz corresponding to a variable Bohr radius,

$$
\Psi_{0}(p ; \alpha)=8 \pi^{1 / 2} \alpha^{-3 / 2}\left(1+p^{2} / \alpha^{2}\right)^{-2} .
$$

Here $\alpha$ is a parameter which characterizes the occupation in the momentum space. In a more refined approach we may take into account that no states below the Fermi momentum are available. The shift of the binding energy as function of the density is shown in Fig. 1. Within a better approximation we take the Fermi function in the zero temperature limit, $f_{e}(p)=\Theta\left(p_{F}-p\right)$, and evaluate the Pauli blocking shift integrating over the wave function $\Psi_{0}(p ; \alpha)$. We obtain

$$
\begin{aligned}
\Delta E_{1}^{\text {Pauli }} & \approx \frac{4 \pi}{(2 \pi)^{3}} \int_{0}^{p_{F}} d p p^{2}\left[p^{2} / \alpha^{2}+1\right] \psi_{0}^{2}(p) \\
& =\frac{4 \alpha^{2}}{\pi}\left[\frac{\left(p_{F}^{2} / \alpha^{2}-1\right) p_{F} / \alpha}{\left(1+p_{F}^{2} / \alpha^{2}\right)^{2}}+\arctan \left(p_{F} / \alpha\right)\right] .
\end{aligned}
$$

We calculate the temperature dependence of the Pauli blocking term by replacing the zero-temperature Fermi function in the interaction term by the finite temperature distribution. It can be seen that the temperature dependence of the Pauli blocking term becomes weak. Even within the variational approach, the densities where the energy 
levels disappear and consequently full ionization occurs, are evidently still too low to explain the observed effects. We include now the Fock term which is of the same order as the Pauli blocking term. Even if the Fock term is not of primary importance for the disappearance of the bound state energy, it has to be included in the total shift of bound and scattering states to be consistent (so-called conserving approximations). In the zero temperature limit we get after some transformations the integral

$$
\Delta E_{1}^{\text {Fock }}=-\frac{64}{\pi^{2}} \int_{0}^{\infty} \frac{p d p}{\left(1+p^{2}\right)^{4}} \int_{0}^{p_{F}} k d k \ln \frac{(p+k)}{|p-k|}=-\frac{4}{3 \pi} p_{F}^{3} \frac{5+3 p_{F}^{2}}{\left(1+p_{F}^{2}\right)^{2}}
$$

which reproduces in the low-density limit the value $\left(-20 \pi n_{e}\right)$ given above in Eq. (7). We may estimate the temperature dependence as above in perturbation theory. For convenience of the numerical procedure in the later variational calculations of the free energy we constructed an interpolation formula between the Boltzmann and the zero temperature limits, i.e. Eqs. (6) and (11), taking into account a few points which we have evaluated numerically,

$$
\Delta E_{1}^{\text {Pauli }}=\frac{4}{\pi}\left\{\frac{p_{F}\left[c(T) p_{F}^{2}-1\right]}{\left[1+c(T) p_{F}^{2}\right]\left(1+p_{F}^{2}\right)}+\arctan \left(p_{F}\right)\right\} .
$$

This is nearly identical to the asymptotic representation except that we had to introduce a fit function $c(T)=(G(T)-1) / 3$ in order to provide the correct derivative at small densities. A similar interpolation can be given for the Fock term

$$
\Delta E_{1}^{\mathrm{Fock}}=-\frac{20 \pi}{g} \ln \left(1+g n_{e}+k n_{e}^{2}+l n_{e}^{3}\right)
$$

with the fit parameters $g=261.65, k=60000, l=334369$. A comparison of the density dependence according to the interpolations introduced above with numerical estimates of the integrals is shown in Fig. 2 for $T=5000 \mathrm{~K}$. The agreement with the data is reasonable for this temperature. In the region of interest $5000 \mathrm{~K}<T<15000 \mathrm{~K}$, the temperature dependence is quite weak. The remaining shifts are smaller and will be neglected here, see also [15, 18, 20, 21].

\section{Ionization equilibrium and thermodynamics in the chemical picture}

We construct the thermodynamic functions of hydrogen by using a chemical approach to the free energy which recently was applied to temperatures between 2000 and 10000 $\mathrm{K}\left[12,22,23,24,13,26,27\right.$. The effects of pressure dissociation, $\mathrm{H}_{2} \rightleftharpoons 2 \mathrm{H}$, and ionization, $\mathrm{H} \rightleftharpoons \mathrm{e}+\mathrm{p}$, are taken into account so that the transition from a molecular fluid at low temperatures and pressures through a partly dissociated, warm fluid at medium temperatures of some thousand Kelvin to a fully ionized, hot plasma above $10000 \mathrm{~K}$ can be explained.

We will not go into the details of the free energy expression used earlier. Here we

take the expression $F_{\mathrm{H}}$ as applied so far [12, 13, 14] and add the contribution due to the energy shifts

$$
F(V, T, N)=V\left(n_{a}+2 n_{m}\right) \Delta E\left(n^{\prime}, T\right)+F_{\mathrm{H}},
$$




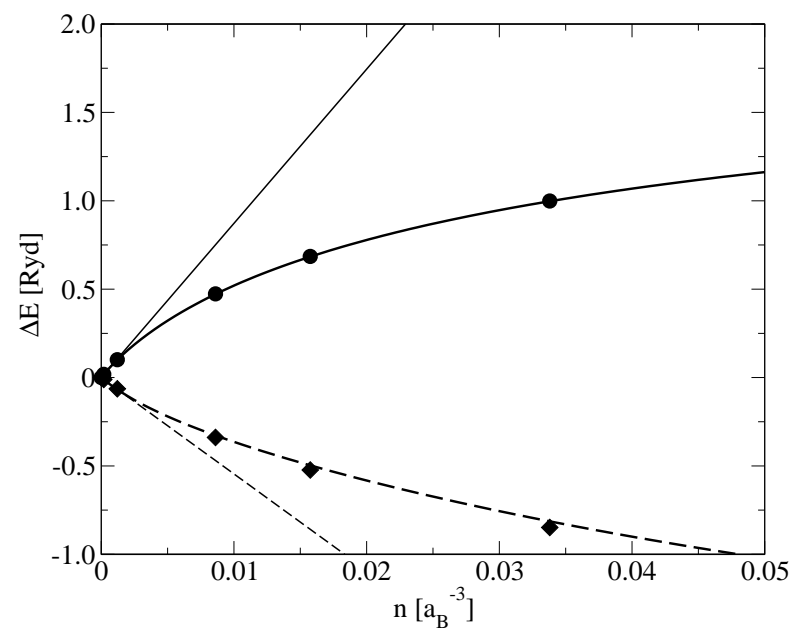

Figure 2. Results of a numerical evaluation of the Pauli shift (dots) and the Fock shift (diamonds) for the temperature $T=5000 \mathrm{~K}$ in comparison to the interpolation formulae (13) and (14) (bold lines) and the Boltzmann approximation (thin lines).

where $n_{a}$ and $n_{m}$ are the number densities of the free atoms and molecules. The shift of the atomic levels is approximated by the sum of Pauli and Fock terms. Furthermore, we assumed that molecules are simply composites of two atoms so that the shifts are additive. We took into account ionisation and dissociation processes. The degrees of ionisation and dissociation defined by [12]

$$
\alpha=\frac{n_{i}}{n_{i}+n_{a}+2 n_{m}}, \quad \beta_{a}=\frac{n_{a}}{n_{i}+n_{a}+2 n_{m}}, \quad \beta_{m}=\frac{2 n_{m}}{n_{i}+n_{a}+2 n_{m}},
$$

are the variational parameters of our problem. The free energy has to be minimized with respect to them. We note that $\beta_{a}$ is the relative amount of protons bound in atoms and $\beta_{m}$ the relative amount bound in molecules. Due to the balance relation for the total proton density we have the simplex relation $\alpha+\beta_{a}+\beta_{m}=1$. Atoms appear only in a rather narrow region of the temperature - density plane. The density dependence of the degrees is represented in Fig. 3. We calculated the chemical equilibrium by means of a numerical variational procedure based on direct minimization of the free energy [25]. We prefer here the minimization of the free energy as compared to the Saha approach because it finds all existing minima, including those at the boundaries. The transition density is between $10^{23}$ and $10^{24}$ protons $/ \mathrm{cm}^{3}$. This means we are in the region around $0.5 \mathrm{~g} / \mathrm{cm}^{3}$ for hydrogen and $1 \mathrm{~g} / \mathrm{cm}^{3}$ for deuterium. The transition to full ionization is rather soft. In principle, all thermodynamic functions may be calculated from the free energy (15) by derivatives. Examples of our results for the pressure and the relative pressure (in relation to the reference pressure of a fully ionized plasma) are shown in Fig. 4. We see a phase transition of first order below $6450 \mathrm{~K}$ and a diffusive transition, defined by wiggles in the relative pressure $p /\left(n_{p} k_{B} T\right)$ up to $20000 \mathrm{~K}$. 


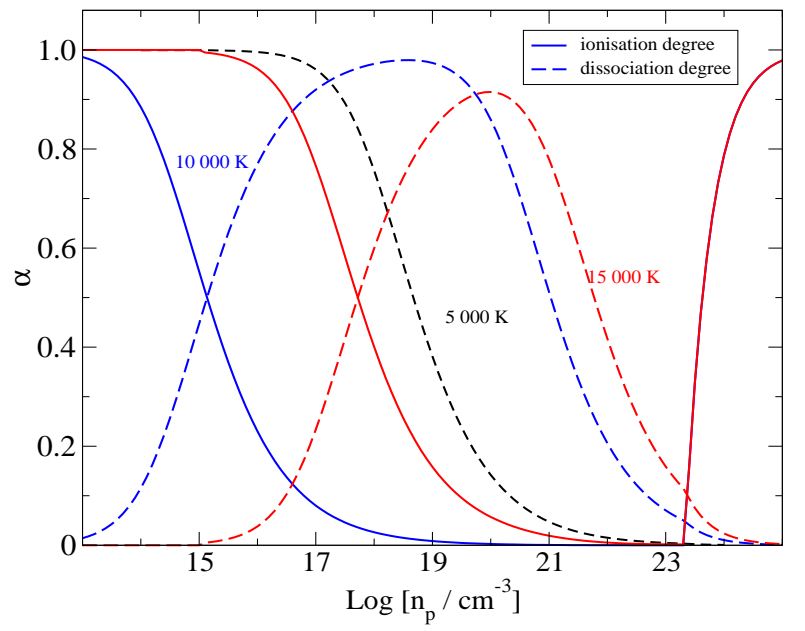

Figure 3. Degree of ionization and degree of dissociation at $T=5000,10000 \mathrm{~K}$ and $15000 \mathrm{~K}$ as a function of the total density of protons.
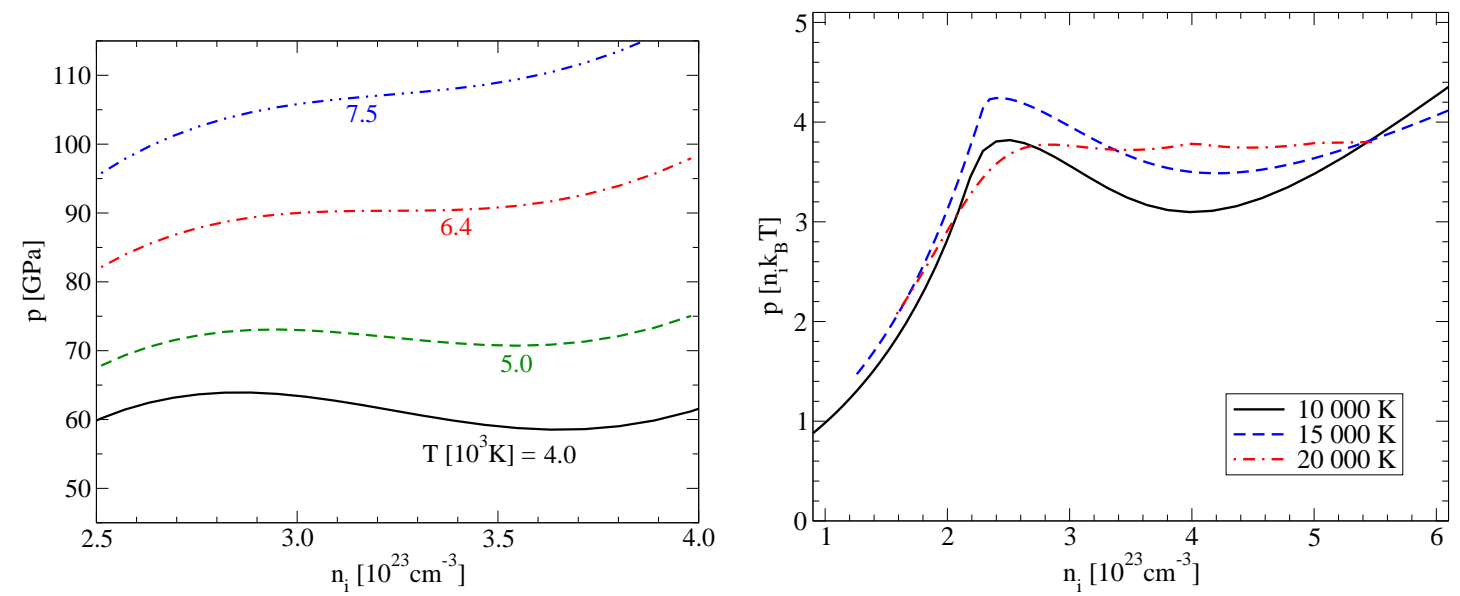

Figure 4. Pressure isotherms (left) and isotherms of the relative pressure (right).

\section{Discussion and Conclusions}

In order to understand the transition to metal-like conductivity in hydrogen at high pressures, we studied the role of Pauli blocking effects. We calculated the Pauli and Fock energy shifts by solving effective Schrödinger equations for strongly correlated systems. The ionization and dissociation equilibria were treated within a chemical approach. We have shown that Pauli blocking effects have a strong influence on the ionization equilibria and the character of the transition in the high pressure region. We presented explicit calculations of the ionization and dissociation equilibria from low to high densities in the region $4000 \mathrm{~K}<T<20000 \mathrm{~K}$. An even more detailed description should take into account higher order contributions to the shifts [20].

The transitions to highly conducting states occur at densities around $3 \cdot 10^{23}$ protons $/ \mathrm{cm}^{3}$. The corresponding pressures are in the region of $(0.8-1.2) \cdot 10^{11} \mathrm{~Pa}$, i.e. around a Mbar. The first order phase transitions are softer than observed in earlier 
work. In the new theory, the first order transition appears only at $T<6450 \mathrm{~K}$, however a diffuse phase transition detected by wiggles of the relative pressure remains up to $20000 \mathrm{~K}$.

We acknowledge helpful discussions with Wolf D. Kraeft and financial support from the DFG funded SFB 652.

\section{References}

[1] W. Ebeling, W.-D. Kraeft, D. Kremp, Theory of Bound States and Ionization Equilibrium (Akademie Verlag, Berlin, 1976).

[2] W.-D. Kraeft, D. Kremp, W. Ebeling, G. Röpke, Quantum Statistics of Charged Particle Systems (Plenum Press, New York, 1986).

[3] D. Kremp, M. Schlanges, W.-D. Kraeft, Quantum statistics of nonideal plasmas (Springer, Berlin, 2005).

[4] E. G. Brovman, Yu. Kagan, Uspekhi Fiz. Nauk 112, 369 (1974).

[5] W. Ebeling, Physica A 130, 587 (1985).

[6] W. Ebeling, W. Richert, Phys. Stat. Sol. (b) 128, 467 (1985); Phys. Lett. A 108, 80 (1985).

[7] D. Saumon, G. Chabrier, Phys. Rev. Lett. 62, 2397 (1989); Phys. Rev. A 46, 2084 (1992).

[8] L. B. Da Silva et al., Phys. Rev. Lett. 78, 483 (1997).

[9] S. T. Weir, A. C. Mitchell, and W. J. Nellis, Phys. Rev. Lett. 76, 1860 (1996).

[10] G. W. Collins et al., Science 281, 1178 (1998).

[11] V. Fortov et al., Phys. Rev. Lett. 99, 185001 (2007).

[12] D. Beule, W. Ebeling, A. Förster, H. Juranek, S. Nagel, R. Redmer, G. Röpke, Phys. Rev. B 59, 14177 (1999).

[13] D. Beule et al., Contrib. Plasma Phys. 39, 21 (1999).

[14] W. Ebeling, H. Hache, H. Juranek, R. Redmer, G. Röpke, Contr. Plasma Phys. 45, 160 (2005).

[15] G. Röpke, K. Kilimann, D. Kremp, W.-D. Kraeft, Phys. Lett. A 68, 329 (1978).

[16] G. Röpke et al., Phys. Stat. Sol. (b) 88, K59 (1978); R. Zimmermann et al., Phys. Stat. Sol. (b) 90, 175 (1978).

[17] W. Ebeling, R. Redmer, H. Reinholz, G. Röpke, Contrib. Plasma Phys. 48, 670 (2008).

[18] G. Röpke et al., Phys. Stat. Sol. (b) 100, 215 (1980); G. Röpke et al., Nucl. Phys. A 399, 587 (1983).

[19] H. C. Graboske, D. J. Harwood, F. J. Rogers, Phys. Rev. 186, 210 (1969).

[20] S. Arndt, W.-D. Kraeft, J. Seidel, Phys. Stat. Sol. (b) 194, 601 (1996).

[21] R. Redmer, Phys. Rep. 282, 35 (1997).

[22] A. Bunker, S. Nagel, R. Redmer, G. Röpke, Phys. Rev. B 56, 3094 (1997); Contrib. Plasma Phys. 37, 115 (1997).

[23] W. Stolzmann, W. Ebeling, Phys. Lett. A 248, 242 (1998).

[24] H. Juranek, R. Redmer, J. Chem. Phys. 112, 3780 (2000).

[25] W. Ebeling, H. Hache, M. Spahn, Eur. Phys. J. D 23, 265 (2003).

[26] D. Beule, W. Ebeling, A. Förster, H. Juranek, R. Redmer, G. Röpke, Phys. Rev. E 63, 060202(R) (2001).

[27] W. Ebeling, A. Förster, V. E. Fortov, V. K. Gryaznov, A. Ya. Polishchuk, Thermophysical Properties of Hot Dense Plasmas (Teubner Verlag, Stuttgart and Leipzig, 1991). 